\title{
Crystal Surface/Liquid Crystal Interfacial Polymerisation: Preparation of Helical $\pi$-Conjugated Polymer on Mineral Crystal
}

\author{
Yuki Kudo, Kohsuke Kawabata, Hiromasa Goto* \\ Division of Materials Science, Faculty of Pure and Applied Sciences, \\ University of Tsukuba, Tsukuba, Ibaraki 305-8573, Japan \\ E-mail: gotoh@ims.tsukuba.ac.jp (H. Goto)
}

Keywords: crystal surface; electrochemical polymerisation, interfacial polymerisation; liquid crystal; helical, liquid crystal, pyrite

\begin{abstract}
Crystal surface/liquid crystal interfacial polymerisation was developed. Poly(3,4-ethylenedioxythiophene) (PEDOT, EDOT $=$ ethylene dioxythiophene) and poly(EDOT-fluorene-EDOT) were prepared in a cholesteric liquid crystal electrolyte solution on the surface of pyrite. Polymerisation in liquid crystal produces polymers showing fingerprint structure through transcription of molecular aggregation in the polymerisation process. Surface structures of the polymers were observed with circular polarised differential interference contrast microscopy (C-DIM) and scanning electron microscopy (SEM). The polymerisation reaction proceeds interface between liquid crystal and pyrite surface. The polymers thus prepared in liquid crystal on the pyrite shows fingerprint structure on steps structure of pyrite. This is a first report of liquid crystal interfacial electrochemical polymerisation on a natural mineral crystal.
\end{abstract}

\section{Introduction}

Electro-active conjugated polymers show excellent performances, such as electrochromism with beautiful colours [1]. We have developed electrochemical polymerisation in liquid crystal. The electrochemical polymerisation has been carried out between sandwiched indium-tin-oxide (ITO) coated glass electrodes in liquid crystal electrolyte solution containing monomer by applying voltage to give polymers on the ITO, which we originally developed previously [2]. Polymers prepared in liquid crystal shows liquid crystal like order because of transcription of liquid crystal order during the polymerisation. The polymer thus prepared shows fingerprint surface structure and chiroptical activity, which is derived from chiral aggregation of cholesteric liquid crystal. Furthermore, circular dichroism and optical rotation of the resultant polymers can be controlled with electrochemical doping-dedoping. This function is referred to as "electro-chiroptic effect".

In this research, we conducted electrochemical polymerisation on pyrite in place of ITO. Pyrite shows beautiful steps structure on the surface. Pyrite can be used as a functional electrode due to semiconducting property [3].

We conduct interfacial electrochemical polymerisation between liquid crystal and pyrite as a mineral crystal. 3,4-Ethylene dioxythiophene derivatives as monomers are employed in this research. A trimer of 3,4-ethylene dioxythiophene (terEDOT, EEE) has been synthesised [4,5].

In the present study, we synthesise poly(3,4-thylenedioxythiophene) (PEDOT) from terEDOT (prepared by Grignard reaction) [5], and poly(EDOT-fluorene-EDOT, EFE) [6,7] in cholesteric liquid crystal on pyrite as a mineral crystal electrode. This is a first report of preparation of helical $\pi$-conjugated polymers on mineral crystal. Combination of chiroptical-electroactivity and $p-n$ junction between the organic conjugated polymer (p-type semiconductor) and pyrite (n-type semiconductor) has possibility for realization of organic/metal semi-conductor devices having optical activity. This preparation method can be referred to as "crystal surface/liquid crystal interfacial polymerisation". 
Table 1. Constituents of cholesteric liquid crystal electrolyte solution and electrode (pyrite, anode)

Supporting salt
4.9 mg (1.1 x10

\section{Material and Method}

A trimer of 3,4-ethylene dioxythiophene (EDOT-EDOT-EDOT, EEE).

EEE was prepared with $\mathrm{Pd}\left(\mathrm{PPh}_{3}\right)_{4}[\mathrm{Pd}(0)]$ by previously reported method [5].

2,7-bis(2,3-dihydrothieno[3,4-b][1,4]dioxin-5-yl)-9H-fluorene (EDOT-Fluorene-EDOT, EFE)

A solution of 2-tributylstannyl-3,4-ethylenedioxythiophene (1.91 g, $4.4 \mathrm{mmol})$ [9], 2,7-dibromofluorene $(0.70 \mathrm{~g}, 2.2 \mathrm{mmol})$ in toluene $(7 \mathrm{~mL})$ was stirred for $5 \mathrm{~min}$ at $\mathrm{rt}$. Then, $\mathrm{Pd}\left(\mathrm{PPh}_{3}\right)_{4}(0.050 \mathrm{~g}, 0.043 \mathrm{mmol})$ was added to the solution and stirred for $10 \mathrm{~h}$ at $80{ }^{\circ} \mathrm{C}$. The crude product was purified with column chromatography $\left(\mathrm{CHCl}_{3}\right.$ /hexane $\left.=2 / 1\right)$. Recrystallisation from $\mathrm{CHCl}_{3} /$ hexane followed by evaporation, and dry under reduce pressure afforded pale green product. $\mathrm{Y}=48 \%$ (0.46g, $1.0 \mathrm{mmol}) .{ }^{1} \mathrm{H}$ NMR $\left(270 \mathrm{MHz}, \mathrm{CDCl}_{3}\right): \delta=3.94$ (s, 2H, 9-H(fluorene)), 4.30 (m, $\left.8 \mathrm{H},-\mathrm{O}-\mathrm{C}_{2} \mathrm{H}_{4}-\mathrm{O}-\right) 6.30$ (s, 2H, 5- $\mathrm{H}$ (thiophene)), 7.72 (m, 4H, 3,4,5,6-H(fluorene)), 7.89 (m, 2H, $1,8-H$ (fluorene)). 


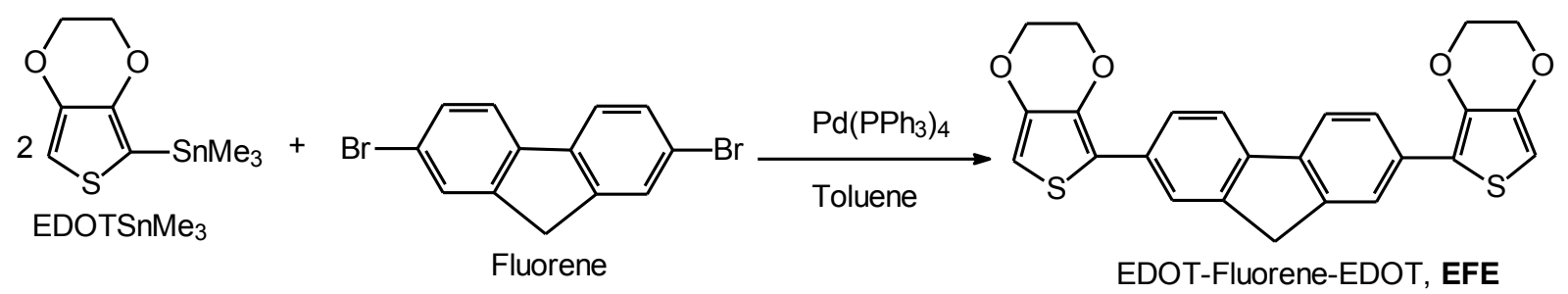

Scheme 1. Synthesis for EFE.

Optical texture observations were carried out using an ECLIPS LV 100 high-resolution polarising microscope (Nikon) with a LU Plan Fluor lens, a CFIUW lens, and a circular polarised differential interference contrast unit (Nikon). SEM observations were carried out using a JEOL JSM-521

\section{Experiment}

Preparation of cholesteric liquid crystal electrolyte solution

First, a cholesteric liquid crystal electrolyte solution containing monomer, tetrabutylammonium perchlorate (TBAP), $n$-hexyl-cyanobiphenyl (6CB), and cholesteryl pelargonate $(\mathrm{C}-\mathrm{Pe})$ was prepared. The chemical structures and functions of constituents of the cholesteric liquid crystal electrolyte solution are summerised in Table 1.6CB is a matrix liquid crystal showing nematic phase. Addition of small amount of chiral inducer C-Pel to the nematic liquid crystal produces formation of cholesteric liquid crystal having helical structure. This is not phase transition. Fundamentally, cholesteric and nematic liquid crystals are the same category in liquid crystal. Distance between two stripes of cholesteric fingerprint structure corresponds to helical half-pitch (half-turn).
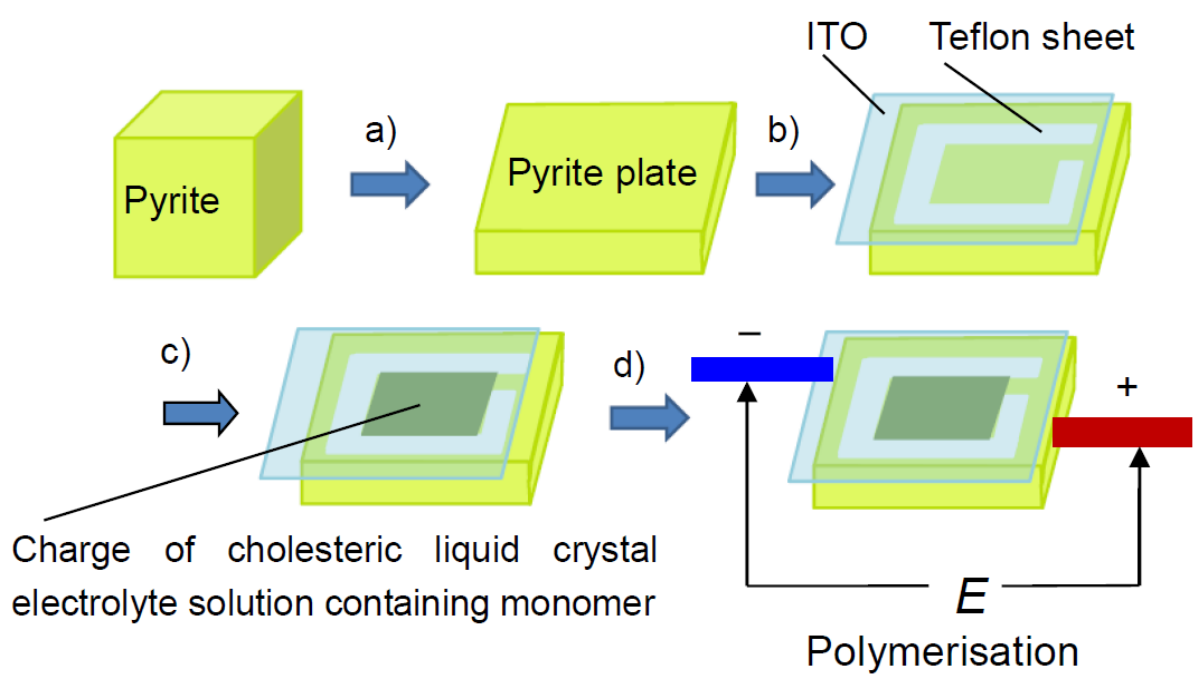

Figure 1. Assembly of sandwich cell with pyrite as anode, and electrochemical polymerisation. (a): Preparation of a thin pyrite plate. (b): Teflon sheet is sandwiched between pyrite and ITO glass.

(c): Injection of liquid crystal electrolyte solution in the cell gap with capillary technique.

(d): Electrochemical polymerisation by applying voltage. 

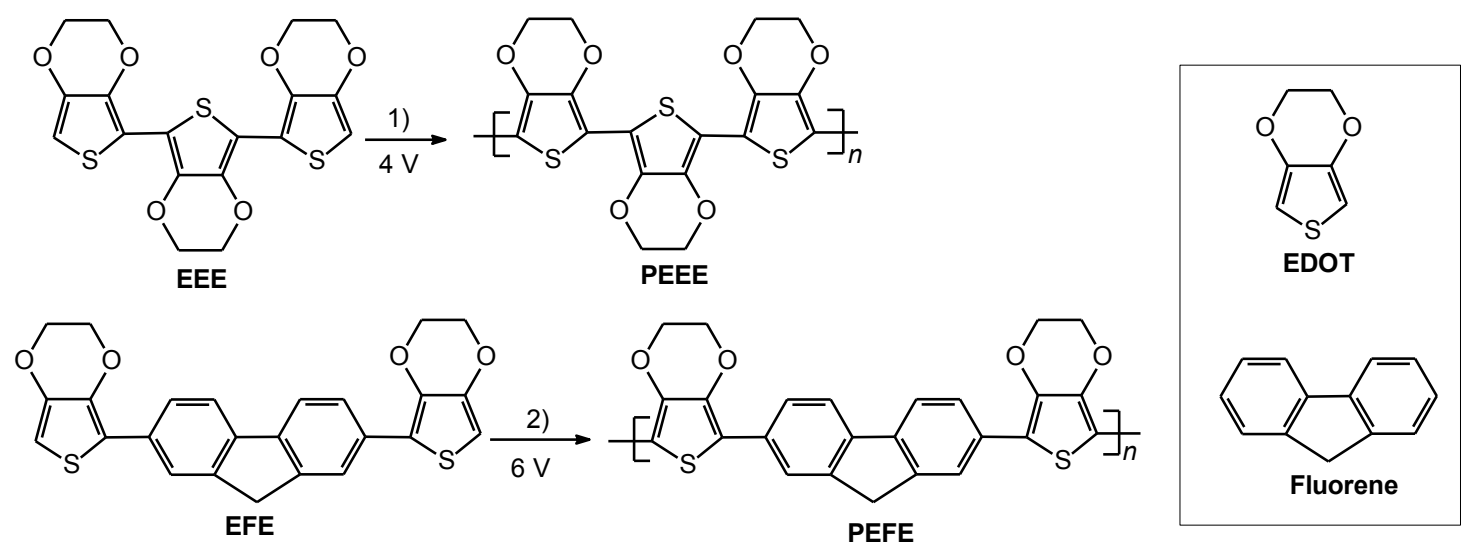

Scheme 2. Electrochemical polymerisation in cholesteric liquid crystal electrolyte. 1), 2): TBAP, $6 \mathrm{CB}$, cholesteryl pelargonate.

\section{Surface structure of pyrite}

Fig. 2a displays entire image of a pyrite crystal as an example, showing gold colour reflection. Figs. 2(b,c) show SEM images of the pyrite surface. High magnification image confirms step-structure of the pyrite.

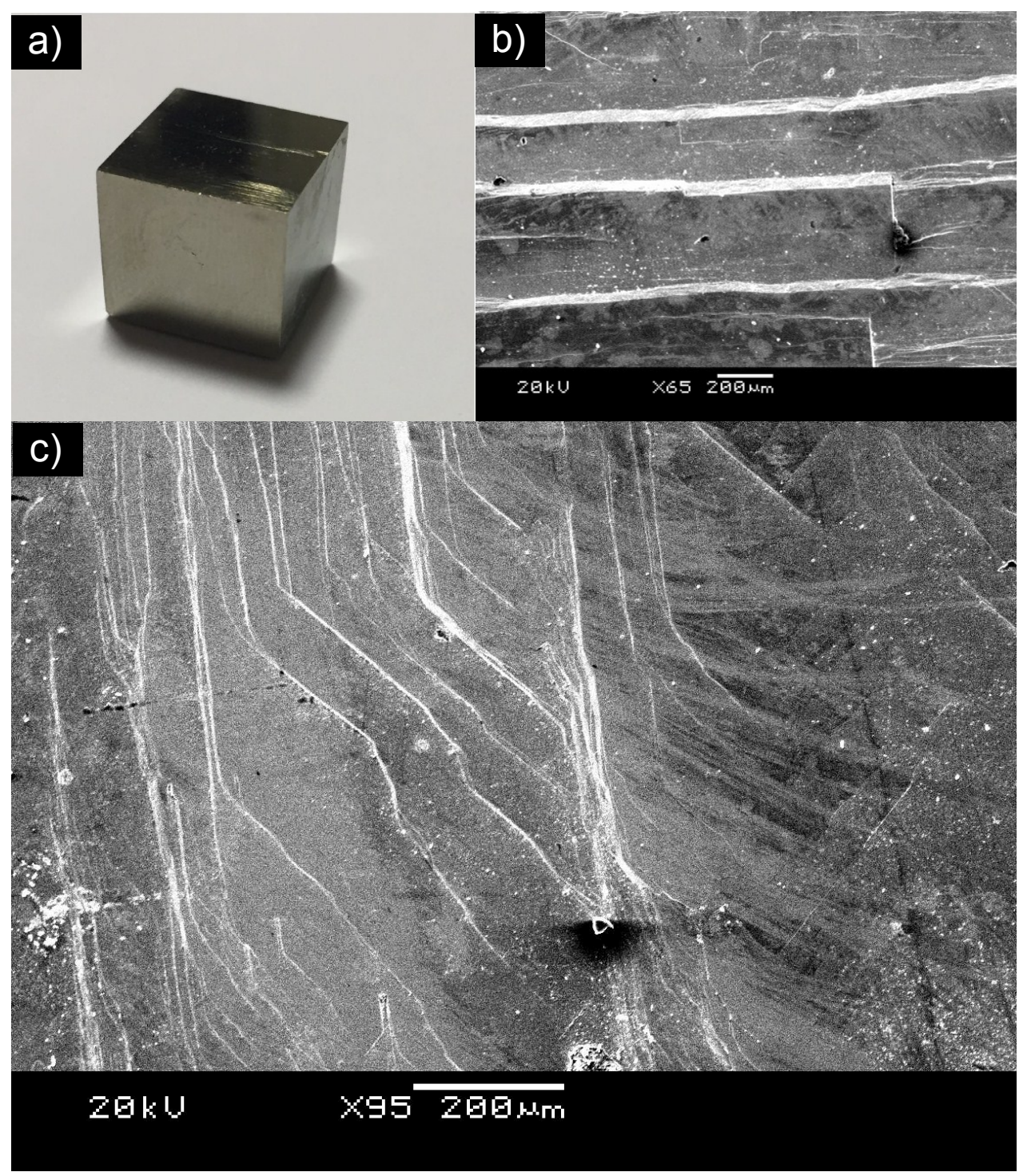

Figure 2. (a): A crystal of pyrite. (b): Scanning electron microscopy (SEM) image of surface of pure pyrite. (c): Low magnification image of surface of pure pyrite. 
Crystal surface/liquid crystal interfacial electrochemical polymerisation

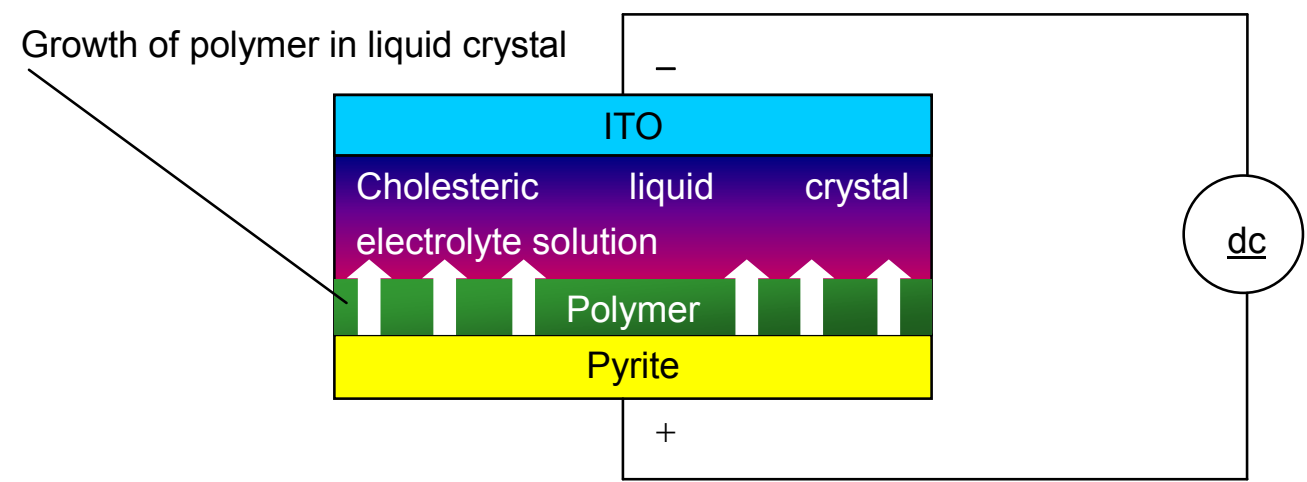

Figure 3. Crystal surface/liquid crystal interfacial electrochemical polymerisation.
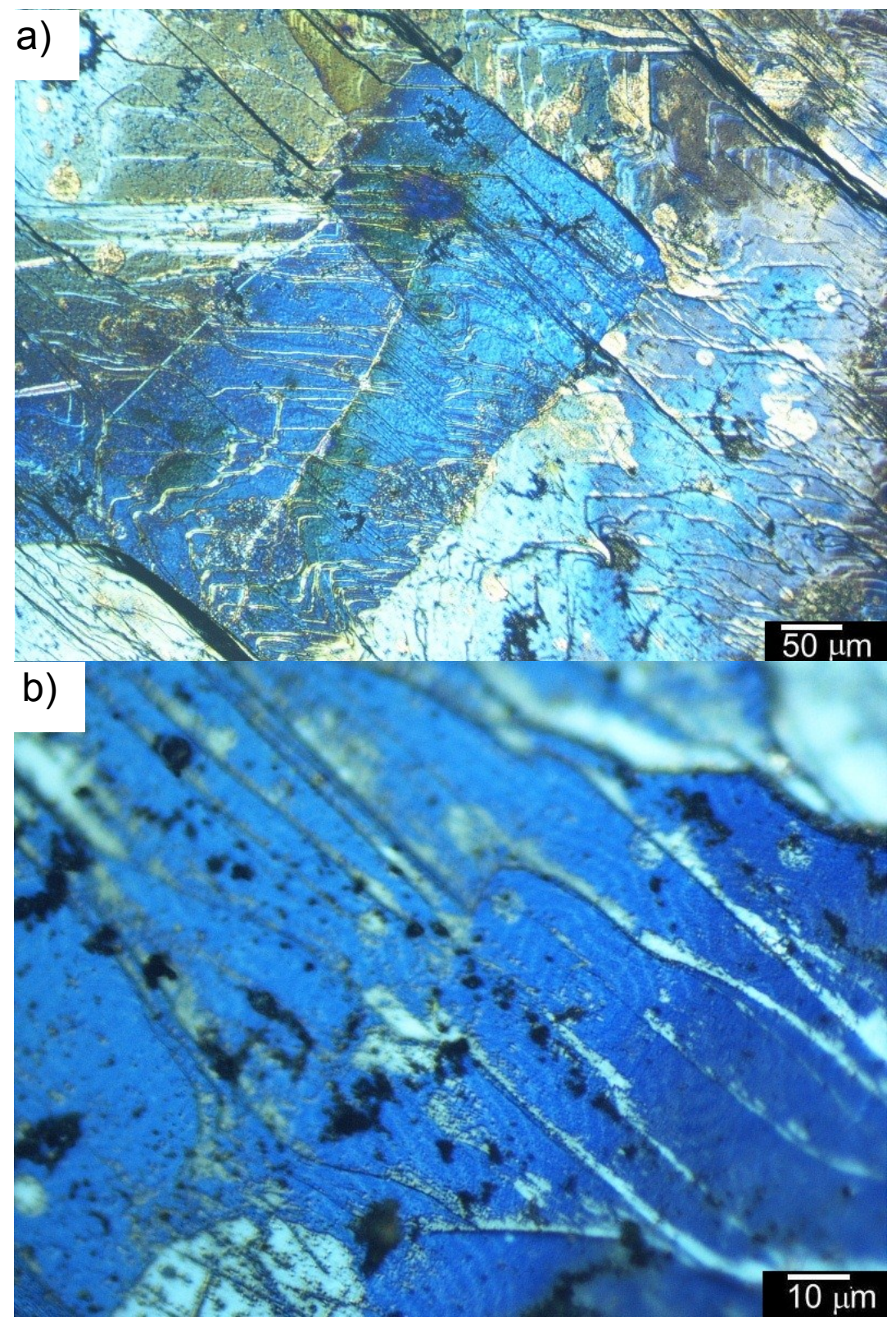

Figure 4. Circular polarised differential interference contrast microscopy (C-DIM) images of poly(EDOT-EDOT-EDOT, poly(terEDOT)) (PEEE) deposited on pyrite. (a): Low magnification image. (b): high magnification image. 
Electrochemical oxidative polymerisation was carried out. We employed terEDOT (abbreviated as EEE), and EDOT-fluorene-EDOT (EFE) as monomers. Direct current (dc) voltage was applied across the cell (Fig. 1d). Scheme 2 shows polymerisation reaction. Electrochemical polymerisation occurs at the pyrite surface as an anode. Then, the surface was washed with large volume of methanol, and dried.

The resultant polymers from EEE and EFE are abbreviated as PEEE (poly(EDOT-EDOT-EDOT, poly(terEDOT)) and PEFE (poly(EDOT-fluorene-EDOT). The crystal surface/liquid crystal interfacial electrochemical polymerisation cell is illustrated in Fig. 3.

\section{Results and Discussion}

\section{Surface structure}

Surface observation with circular polarised differential interference contrast microscopy (C-DIM) was carried out. Fig. 4 shows the C-DIM image of PEEE deposited on the pyrite. Fingerprint structure on the surface steps of the pyrite was confirmed, although low magnification image can not indicate it clearly (Fig. 4a). Fig. 5 shows scanning electron microscopy (SEM) image of PEEE. Magnification image evaluates that PEEE shows fine rope-like structure drawing fingerprint structure.

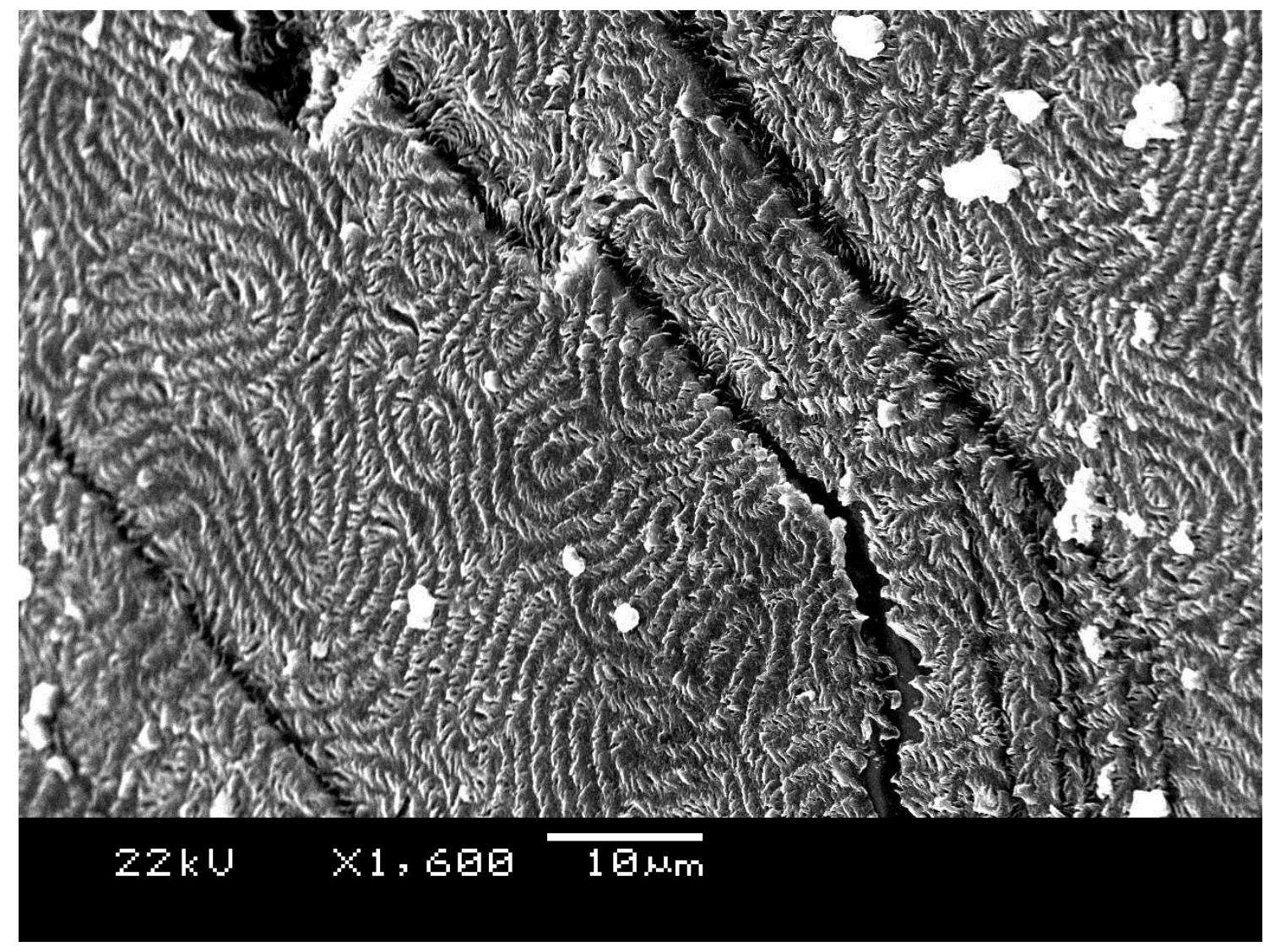

Figure 5. Scanning electron microscopy (SEM) image of PEEE deposited on the pyrite.

C-DIM images of PEFE are displayed in Fig. 6. PEFE shows no rope-like structure. The PEFE layer is well deposited on the pyrite. The magnification image demonstrated fingerprint texture of PEFE on the pyrite surface.

\section{Mechanism}

The rope like texture and fingerprint texture are formed through transcription of molecular aggregation of cholesteric liquid crystals during the polymerisation process. 


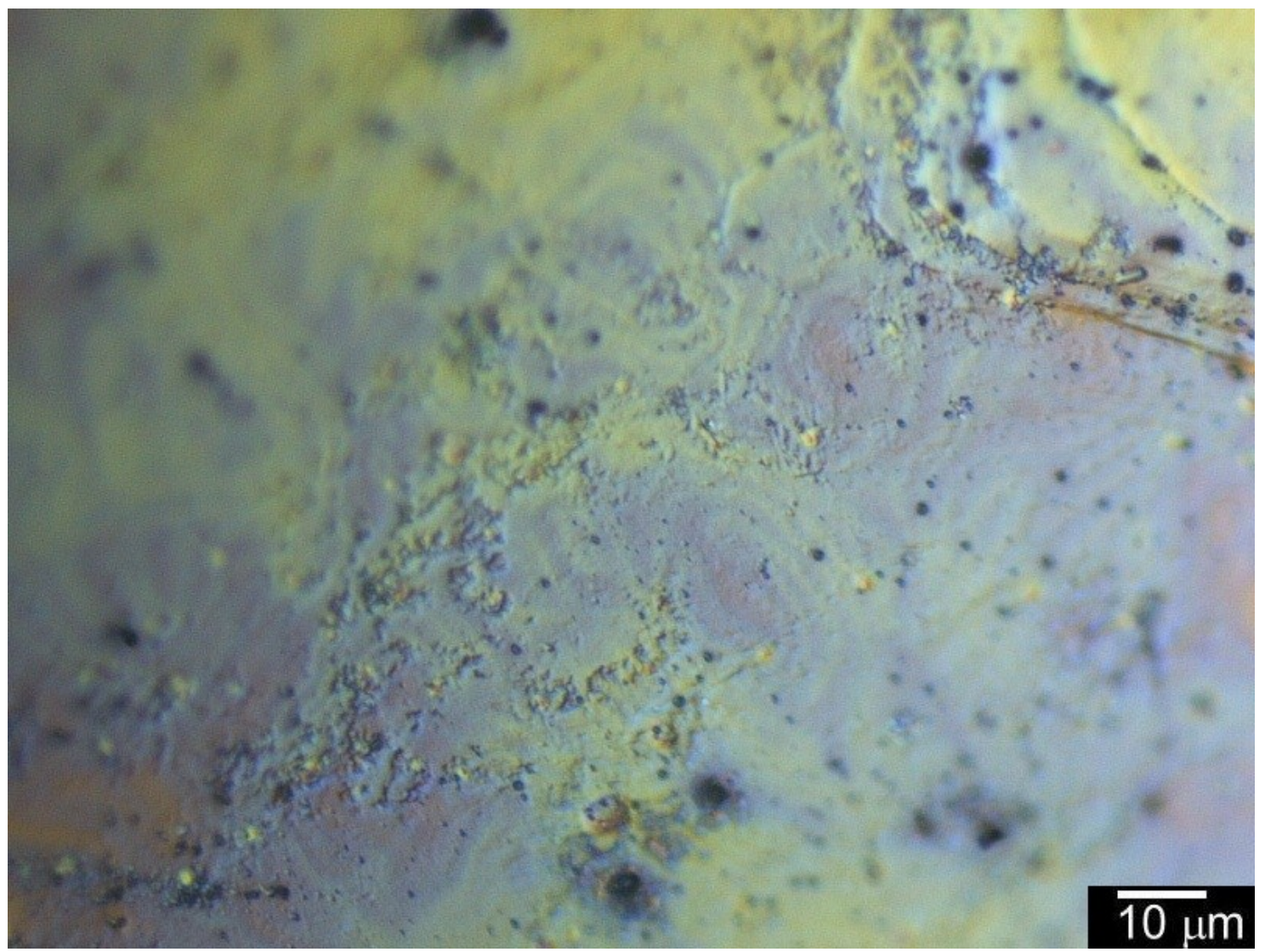

Figure 6. C-DIM images of poly(EDOT-fluorene-EDOT) (PEFE).

The surface steps structure of the substrate [10] provides mechanical steps of the polymers. Plausible structure for PEEE is shown in Fig. 7. Polymerisation was occurred in the cholesteric liquid crystal matrix with helical structure from anode side of the pyrite. The polymer was propagated with transcription of molecular aggregation form of the helical structure in the cholesteric liquid crystal. Resultant polymer shows helical structure derived from the liquid crystal matrix, and steps structure derived from pyrite surface. Although PEFE shows no rope like structure, fingerprint structure is observable with the C-DIM. Difference of the morphologies between PEEE and PEFE can be derived from difference of crystallinity and molecular packing form.

\section{Conclusions}

Electrochemical polymerisation in cholesteric liquid crystal electrolyte solution was successfully carried out on pyrite. The resultant polymers show fingerprint structure on the steps of pyrite in micro-level. This method is combination of liquid crystal transcription polymerisation and crystal surface polymerisation. Note that this report can be a first example of preparation of helical $\pi$-conjugated polymer with chiroptical activity on a mineral crystal. A p-n junction between the helical conjugated polymer (p-type) and pyrite (n-type) can be expected for applications as organic/inorganic semiconductor devices.

\section{Acknowledgments}

We would like to thank Chemical Analysis Division, Research Facility Centre for Science and Technology, University of Tsukuba, and Glass Work Shop of University of Tsukuba. This research was supported by the Japan Society for the Promotion of Science, Grant-in-Aid for Scientific Research, 25410218, and Tsukuba Research Centre for Interdisciplinary Materials Science (TIMS). 


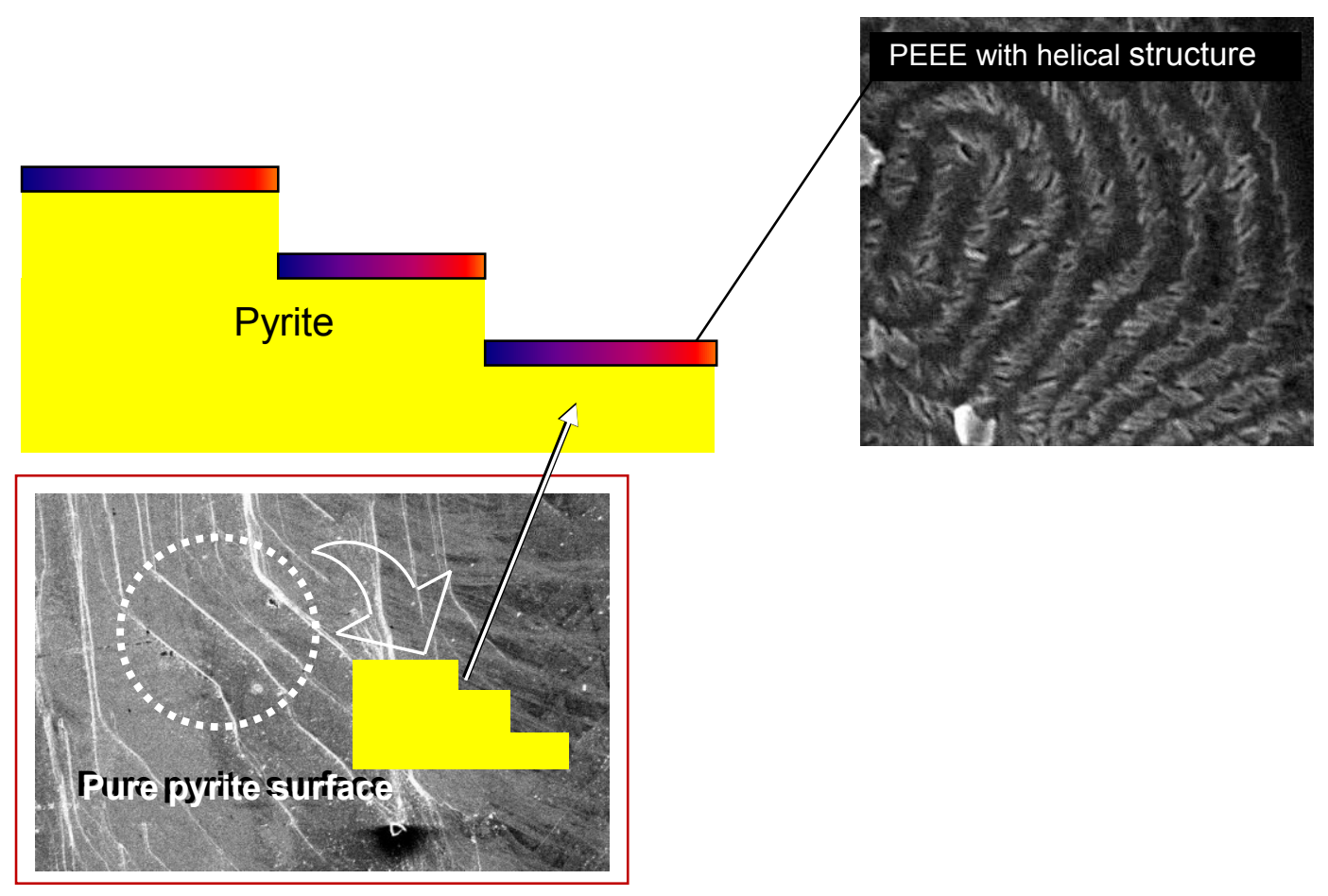

Figure 7. Structure of PEEE prepared in cholesteric liquid crystal on the pyrite.

\section{References}

[1] A.A. Argun et al., Multicolored electrochromism in polymers: structures and devices, Chem. Mater. 16 (2004) 4401-4412.

[2] H. Goto, Lab. Res Note, U. Tsukuba, University of Tsukuba, 24 (2000) 2416. http://www.ims.tsukuba.ac.jp/ gotoh_lab/Note\%20Goto.pdf

[3] H. Horita, Semiconducting properties of natural pyrite (iii): impurity-concentration dependences and characteristics of d-band, Tohoku University, Departmental Bulletin Paper. 29 (1973) 50-61.

[4] J.-M. Raimundo, P. Blanchard, H. Brisset, S. Akoudad, J. Roncali, Proquinoid acceptors as building blocks for the design of efficient $\pi$-conjugated fluorophores with high electron affinity, Chem. Commun. (2000) 939-940.

[5] H. Goto, Vortex fibril structure and chiroptical electrochromic effect of optically active poly(3,4-ethylenedioxythiophene) (PEDOT*) prepared by chiral transcription electrochemical polymerisation in cholesteric liquid crystal, J. Mater. Chem. 19 (2009) 4914-4921.

[6] K. Kawabata, Master Graduation thesis, University of Tsukuba, March 2010.

[7] A. Wang, K. Kawabata, H. Kawashima, H. Goto, Synthesis of a pyrimidine-based new chiral inducer for construction of cholesteric liquid crystal electrolyte solution and its electrochemical polymerization, and stimulated emission like interference, Polymer. 54 (2013) 3821-3827.

[8] G. Nie, H. Yang, J. Chen, Z. Bai, A novel high-quality electrochromic material from 3,4-ethylenedioxythiophene bis-substituted fluorine, Org. Elect. 13 (2012) 2167-2176.

[9] G. E. Gunbas, A. Durmus, L. Toppare, Could green be greener? novel donor-acceptor-type electrochromic polymers: towards excellent neutral green materials with exceptional transmissive oxidized states for completion of RGB color space, Adv. Mater. 20 (2008) 691-695.

[10]Nakada, Visual observations of crystal growth (in Japanese), Agune Tech Centre, 1995 Tokyo. 